\title{
A Dyadic Wavelet Filtering Method for 2-D Image Denoising
}

\author{
Yonggui Zhu*, Xiaolan Yang \\ School of Science, Communication University of China, Beijing, China. \\ Email: *ygzhu@cuc.edu.cn
}

Received January $11^{\text {th }}, 2011$; revised July $16^{\text {th }}, 2011$; accepted July $24^{\text {th }}, 2011$.

\begin{abstract}
We improve spatially selective noise filtration technique proposed by Xu et al. and wavelet transform scale filtering approach developed by Zheng et al. A novel dyadic wavelet transform filtering method for image denoising is proposed. This denoising approach can reduce noise to a high degree while preserving most of the edge features of images. Different types of images are employed to test in the numerical experiments. The experimental results show that our filtering method can reduce more noise contents while maintaining more edges than hard-threshold, soft-threshold filters, Xu's method and Zheng's method.
\end{abstract}

Keywords: Dyadic Wavelet Transform, Image Edges, Denoisin

\section{Introduction}

Wavelet transform is a multi-resolution representation of a signal or image. It is a powerful tool in several areas of applications like signal processing, image processing, pattern recognition, data compression, commutation, etc. Singularities and irregular structures often carry essential information in signals and images. For example, the discontinuities of the intensity of an image indicate the locations of edges.

The local regularity is characterized by the decay of the wavelet transform amplitude across scales. Signal singularities and image edges can be detected by the dyadic wavelet transform modulus maxima across scales $[1,2]$. In mathematics, singularities are generally measured with Lipschitz exponents. The wavelet theory proves that these Lipschitz exponents can be calculated from the propagating amplitude values of the different modulus maxima across scales.

The original signal or image has singularities whose Lipschitz exponents are greater than or equal to zero, and the noise has singularities whose Lipschitz exponents are less than zero. Thus, the amplitudes of signal or image modulus maxima increase when the scale increases, while the amplitude of noise modulus maxima decrease strongly when the scale increases. By using these properties, the noises can be eliminated from the noised signals or images. The approaches for separating signal and noise in wavelet scale space are proposed by many researchers. For example, the original signal can be extracted from the noisy version by estimating the signal modulus maximum at small scales $[1,2]$. Adaptive Wiener filtering were used to remove noise in signals and images [3-5]. The selective noise filtration technique and adaptive thresholding function in image denoising were developed [6-8]. The scale space filtering algorithms applied to image denoising were also proposed $[9,10]$. In addition, many other novel approaches for image denoising have been presented by some researchers [11-13] recently. In this work, we develop an image denoising approach by improving spatially selective noise filtration technique proposed by $\mathrm{Xu}$ et al. [6] and wavelet transform scale filtering approach given by Zheng et al. [9]. Hard-threshold and soft-threshold filters that were proposed by D. L. Donoho $[14,15]$ are widely used in image denoising processing. We will compare our filtering approach with hard-threshold filtering, soft-threshold filtering, Xu's method and Zheng's method in the numerical experime- nts. Peak-Signal-Noise-Rate (PSNR) and Root-Mean-SquareError (RMSE) are employed to estimate the quality of restored images.

\section{2-D Dyadic Wavelet Transform}

Let $\psi^{(k)}\left(x_{1}, x_{2}\right) \quad(\mathrm{k}=1,2)$ be wavelet functions.

We denote that

$$
\left.\begin{array}{l}
\psi_{2^{j}}^{(1)}\left(x_{1}, x_{2}\right)=\frac{1}{2^{j}} \psi^{(1)}\left(\frac{x_{1}}{2^{j}}, \frac{x_{2}}{2^{j}}\right) \\
\psi_{2^{j}}^{(2)}\left(x_{1}, x_{2}\right)=\frac{1}{2^{j}} \psi^{(2)}\left(\frac{x_{1}}{2^{j}}, \frac{x_{2}}{2^{j}}\right)
\end{array}\right\}
$$


The wavelet transform of $f\left(x_{1}, x_{2}\right) \in L^{2}\left(R^{2}\right)$ at the scale $2^{j}$

Is

$$
\left.\begin{array}{r}
W_{2^{j}}^{(1)} f\left(x_{1}, x_{2}\right)=f * \psi_{2^{j}}^{(1)}\left(x_{1}, x_{2}\right) \\
W_{2^{j}}^{(2)} f\left(x_{1}, x_{2}\right)=f * \psi_{2^{j}}^{(2)}\left(x_{1}, x_{2}\right)
\end{array}\right\}
$$

The set of functions

$$
W f=\left\{W_{2^{j}}^{(1)} f\left(x_{1}, x_{2}\right), W_{2^{j}}^{(2)} f\left(x_{1}, x_{2}\right)\right\}_{j \in \mathrm{Z}}
$$

is called 2-D dyadic wavelet transform of $f\left(x_{1}, x_{2}\right)$. The Fourier transforms of $\psi^{(1)}\left(x_{1}, x_{2}\right)$ and $\psi^{(2)}\left(x_{1}, x_{2}\right)$ are $\hat{\psi}^{(1)}\left(\omega_{1}, \omega_{2}\right)$ and $\hat{\psi}^{(2)}\left(\omega_{1}, \omega_{2}\right)$

We suppose that $\chi^{(1)}\left(x_{1}, x_{2}\right), \chi^{(2)}\left(x_{1}, x_{2}\right)$ are reconstructed wavelet functions. If their Fourier transforms satisfy

$$
\sum_{j=-\infty}^{+\infty}\left(\begin{array}{l}
\hat{\psi}^{(1)}\left(2^{j} \omega_{1}, 2^{j} \omega_{2}\right) \hat{\chi}^{(1)}\left(2^{j} \omega_{1}, 2^{j} \omega_{2}\right) \\
+\hat{\psi}^{(1)}\left(2^{j} \omega_{1}, 2^{j} \omega_{2}\right) \hat{\chi}^{(1)}\left(2^{j} \omega_{1}, 2^{j} \omega_{2}\right)
\end{array}\right)=1
$$

Then $f\left(x_{1}, x_{2}\right)$ can be reconstructed from their dyadic wavelet transform i.e.

$$
f\left(x_{1}, x_{2}\right)=\sum_{j=-\infty}^{+\infty}\left(\begin{array}{l}
W_{2^{j}}^{(1)} f * \chi_{2^{j}}^{(1)}\left(x_{1}, x_{2}\right) \\
+W_{2^{j}}^{(1)} f * \chi_{2^{j}}^{(1)}\left(x_{1}, x_{2}\right)
\end{array}\right)
$$

Because of the limitation of image's resolution, we introduce a smoothing function $\phi\left(x_{1}, x_{2}\right)$

Whose Fourier transform satisfies

$$
\left|\hat{\phi}\left(\omega_{1}, \omega_{2}\right)\right|^{2}=\sum_{j=1}^{+\infty}\left(\begin{array}{l}
\hat{\psi}^{(1)}\left(2^{j} \omega_{1}, 2^{j} \omega_{2}\right) * \hat{\chi}^{(1)}\left(2^{j} \omega_{1}, 2^{j} \omega_{2}\right) \\
+\hat{\psi}^{(2)}\left(2^{j} \omega_{1}, 2^{j} \omega_{2}\right) * \hat{\chi}^{(2)}\left(2^{j} \omega_{1}, 2^{j} \omega_{2}\right)
\end{array}\right)
$$

We define the smoothing operator $S_{2^{j}}$ by

$$
\left.\begin{array}{c}
S_{2^{j}} f\left(x_{1}, x_{2}\right)=f * \phi_{2^{j}}\left(x_{1}, x_{2}\right) \\
\phi_{2^{j}}\left(x_{1}, x_{2}\right)=\frac{1}{2^{j}} \phi\left(\frac{x_{1}}{2^{j}}, \frac{x_{2}}{2^{j}}\right)
\end{array}\right\}
$$

The wavelet transform between the scales 1 and

$$
2^{J}\left\{W_{2^{j}}^{(1)} f\left(x_{1}, x_{2}\right), W_{2^{j}}^{(2)} f\left(x_{1}, x_{2}\right)\right\}_{1 \leq j \leq J}
$$

provides the details that are in $S_{1} f\left(x_{1}, x_{2}\right)$ but that have lost in $S_{2^{j}} f\left(x_{1}, x_{2}\right)$.

Mallat [1] has given the fast algorithm for the discrete dyadic wavelet transform. The fast dyadic wavelet transform can also be calculated with a filter bank algorithm called the algorithm $a^{\prime}$ trous proposed by Holschneider, Kronland-Martinet, Morlet and Tchamitchian [16]. In this paper, we use $a^{\prime}$ trous algorithm to reconstruct the image.

\section{Dyadic Wavelet Transform Filtering Algorithm}

In recent years, some denoising techniques based on the wavelet transform have been studied by many authors $[2,6,12,17]$. The edge modulus maxima can be distinguished from noise modulus maxima by analyzing the singularity properties of wavelet transform domain maxima of a signal or image across scales [2]. Y. Xu [6] developed wavelet transform domain filters based on the direct spatial correlation of the wavelet transform at several adjacent scales. Y. Zheng [9] proposed a wavelet transform scale filtering algorithm by using the properties of signal and noise modulus maxima across large scales. Our approach relies on the variations of the dyadic wavelet transform data across all scales to remove noises rather than extracting edges directly.

For a 2-D image, the discrete sampling of

$$
\begin{aligned}
& \left\{W_{2^{j}}^{(k)} f\left(x_{1}, x_{2}\right)\right\}_{1 \leq j \leq J} \text { is given by } \\
& W_{2^{j}}^{(k)^{d}} f\left(k_{1}, k_{2}\right)=\left.W_{2^{j}}^{(k)} f\left(x_{1}, x_{2}\right)\right|_{\left(x_{1}, x_{2}\right)=\left(k_{1}, k_{2}\right) \in \mathrm{Z}^{2}} 1 \leq j \leq J(7)
\end{aligned}
$$

The discrete coarse smoothed image is denoted by

$$
S_{2^{J}}^{d} f\left(k_{1}, k_{2}\right)=\left.S_{2^{J}} f\left(x_{1}, x_{2}\right)\right|_{\left(x_{1}, x_{2}\right)=\left(k_{1}, k_{2}\right) \in Z^{2}}
$$

In the scale space, the modulus maxima of $W_{2^{j}}^{d(k)} f$ across scales produced by image edges have positive correlation. When the scale $j$ increases, the amplitudes of modulus maxima coeffcients will increase or retain constant. On the contrary, the modulus maxima produced by noises have negative correlation and the amplitudes of their coeffcients decrease as $j$ increases.

Define 2-scale correlation as

$$
\begin{aligned}
& C r 2^{(k)}(m, n)=W c^{(k)}(m, n) * W c^{(k)}(m+1, n), \\
& m=1,2, \cdots, J-1, n=\left(n_{1}, n_{2}\right), 1 \leq n_{1}, n_{2} \leq N,
\end{aligned}
$$

where $W c^{(k)}(m, n)=W_{2^{m}}^{(k) d} f(n)=W_{2^{m}}^{(k) d} f\left(n_{1}, n_{2}\right) . \quad J$ represents the maximum scale of the decomposition.

The 2-scale direct correlation sharpens and enhances major edges while suppressing noise and small features. So comparing the values of $\mathrm{Cr} 2^{(k)}(m, n)$ and $W c^{(k)}(m, n)$ can separate important edges from noise in images. Before comparison, $\mathrm{Cr} 2^{(k)}(m, n)$ needs to be rescaled to $W c^{(k)}(m, n)$ Xu's rescaling scheme is

$$
C r 2^{\prime(k)}(m, n)=C r 2^{(k)}(m, n) \sqrt{P W^{(k)}(m) / P C r 2^{(k)}(m)}
$$

where $P C r 2^{(k)}(m)=\sum_{n} C r 2^{k}(m, n)^{2}$

and $P W^{(k)}(m)=\sum_{n} W c^{(k)}(m, n)^{2}$. 
Zheng et al. use the modulus maxima rescaling method at large scales, and apply the above mentioned rescaling method at small scales. Let $S$ be the upper limit of small scales, assume

$$
\operatorname{Mcr}^{(k)}(m)=\max _{n=\left(n_{1}, n_{2}\right) \in \mathrm{N}^{2}}\left(\left|\operatorname{Cr} 2^{(k)}(m, n)\right|\right), 1 \leq m \leq S
$$

and

$$
M w c^{(k)}(m)=\left|W c^{(k)}(m, \tau)\right|, 1 \leq m \leq S
$$

The modulus maxima rescaling formula as follows:

$$
C r 2^{\prime(k)}(m, n)=C r 2^{(k)}(m, n)\left(\frac{M w c^{(k)}(m)}{M c r^{(k)}(m)}\right), 1 \leq m \leq S
$$

At small scales, noise in the noised image is dominating except some sharp image edges. According to $\mathrm{Xu}$ and Zheng's ideas, if compare $\left|\mathrm{Cr} 2^{\prime(k)}(m, n)\right|$ with $\left|W c^{(k)}(m, n)\right|$ directly, then too much noise will be extracted as edges. To avoid this drawback, we apply the modulus maxima rescaling at all scales and renew the formula (13) as

$$
\begin{aligned}
& C r 2^{\prime(k)}(m, n)=\lambda_{m}^{k} C r 2^{(k)}(m, n)\left(\frac{M w c^{(k)}(m)}{M c r^{(k)}(m)}\right), \\
& m=1,2, \cdots, J-1,
\end{aligned}
$$

where $\lambda_{m}^{(k)}$ is a weight parameter with respect to the scale $\mathrm{m}$.

After rescaling $C r 2^{(k)}(m, n)$ to $W c^{(k)}(m, n)$ for all $\mathrm{m}$ and $\mathrm{n}$, the important edges can be identified in $W c^{(k)}(m, n)$ by comparing the absolute values of $\mathrm{Cr} 2^{\prime(k)}$ and $W c^{(k)}(m, n)$. If $\left|C r 2^{\prime^{(k)}}(m, n)\right| \geq\left|W c^{(k)}(m, n)\right|$ at the point $\tilde{n}=\left(\tilde{n}_{1}, \tilde{n}_{2}\right)$, we retain the value of $W c^{(k)}(m, n)$ at the point $\tilde{n}=\left(\tilde{n}_{1}, \tilde{n}_{2}\right)$. We use a new matrix named $W_{\text {new }}^{(k)}(m, n)$ to represent the retained value, i.e. $W_{\text {new }}^{(k)}(\tilde{m}, \tilde{n})=W c^{(k)}(\tilde{m}, \tilde{n})$. Making comparison at the scale $\mathrm{m}$ for all points $n=\left(n_{1}, n_{2}\right)\left(1 \leq n_{1}, n_{2} \geq N\right)$, we identify $W_{\text {new }}^{(k)}(m, n)$ which represents the most important features of the image edges. We set the values of $C r 2^{(k)}(m, n)$ and $W c^{(k)}(m, n)$ to 0 's at the positions identified and thus obtain a new set of $\operatorname{Cr} 2^{(k)}(m, n)$ and $W c^{(k)}(m, n)$ labelled as $\overline{C r 2^{(k)}(m, n)}$ and $\overline{W c^{(k)}(m, n)}$.

Next we go to rescale and compare $\overline{C r 2^{(k)}(m, n)}$ with $\overline{W c^{(k)}(m, n)}$ and extract the wavelet transform coeffcients that correspond to the next most important features of the image edges. We repeat this process until all major image edges are acquired.
Figure 1 shows the effect of this wavelet transform filtering method at the scale $m=3$ and $\lambda_{m}^{(k)}=m^{3}=27$. In this figure, (a) is the original Lena image, (b) is noised image containing Gaussian white noise with the standard deviation $\sigma=35$. $W c^{(1)}(3, n)$ and $W c^{(2)}(3, n)$ are given in Figures 1(c) and (d), respectively.

$W c^{(1)}(3, n) * W c^{(1)}(4, n)$ and $W c^{(2)}(3, n) * W c^{(2)}(4, n)$

are shown in Figures 1(e) and (f). Figures 1(g) and (h) present $W_{\text {new }}^{(1)}(3, n)$ and $W_{\text {new }}^{(2)}(3, n)$.

By the above mentioned approach, we acquire the filtered dyadic wavelet transform data $\left\{W_{n e w}^{(k)}\right\}_{1 \leq m \leq J-1}$. Let $W_{\text {new }}^{(k)}(J, n)=W^{(k)}(J, n)=W_{2}^{(k) d} f\left(n_{1}, n_{2}\right)$, we can reconstruct the filtered image from the set $\left\{W_{\text {new }}^{(1)}(j, n), W_{\text {new }}^{(2)}(j, n), S_{2^{J}}(n)\right\}_{1 \leq j \leq J}$ where

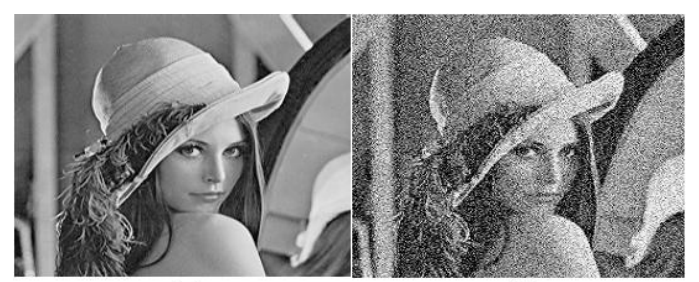

(a)

(b)

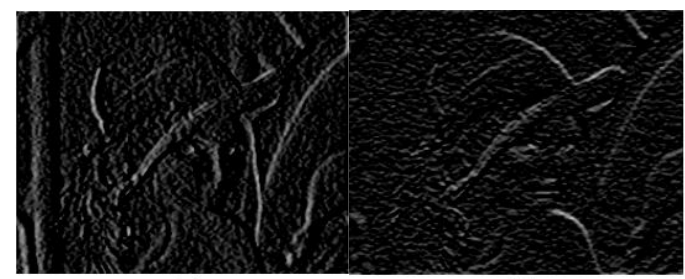

(c)

(d)

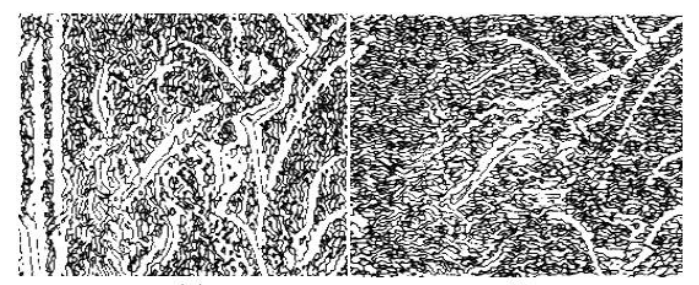

(e) (f)

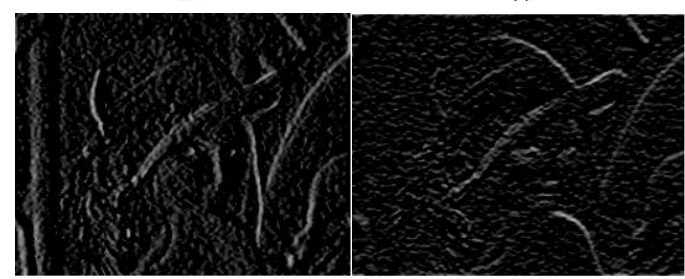

(g)

(h)

Figure 1. The effect of the new wavelet filtering at the scale $m=3$ and $\lambda_{m}^{(k)}=m^{3}=27$. (a) The original Lena image; (b) the noised image; (c) $W c^{(1)}(3, n) ;$ (d) $W c^{(2)}(3, n) ;$ (e) $W c^{(1)}(3, n) * W c^{(1)}(4, n)$; (f) $W c^{(2)}(3, n) * W c^{(2)}(4, n) ;(\mathrm{g})$ $W_{\text {new }}^{(1)}(3, n) ;\left(\right.$ h) $W_{\text {new }}^{(2)}(3, n)$. 
$S_{2^{J}}(n)=S_{2^{J}}^{d} f\left(n_{1}, n_{2}\right)$ through the inverse dyadic wavelet transform. The filtering algorithm is summarized as follows.

\section{Wavelet Transform Filtering Algorithm for Image Denoising}

Step 1. Compute the discrete wavelet transform of noised image $f\left(x_{1}, x_{2}\right)$ and its the lower-frequency smoothed image:

Step 3. Loop for iteration

\{

Loop for the scale $\mathrm{m} \quad(1 \leq m \leq J-1)$

$$
\begin{aligned}
& \operatorname{Mcr}^{(k)}(m)=\max _{n=\left(n_{1}, n_{2}\right) \in N^{2}}\left(\left|C r 2^{(k)}(m, n)\right|\right) \\
& \operatorname{Mwc}^{(k)}(m)=\left|W c^{(k)}(m, \tau)\right|, \text { where }\left|C r 2^{(k)}(m, \tau)\right|=\operatorname{Mcr}^{(k)}(m)
\end{aligned}
$$

Loop for each pixel point $n=\left(n_{1}, n_{2}\right)$

$$
\begin{aligned}
& \left\{W^{(k)}(j, n)\right\}_{1 \leq j \leq J}=\left\{W_{2^{j}}^{(k) d} f\left(n_{1}, n_{2}\right)\right\}_{1 \leq j \leq J} \text { and } \\
& S_{2^{J}}(n)=S_{2^{J}}^{d} f\left(n_{1}, n_{2}\right)
\end{aligned}
$$

Step 2. Initialize:

$$
\begin{aligned}
& W b^{(k)}(m, n)=W c^{(k)}(m, n)=W^{(k)}(m, n), \\
& \text { Filter }^{(k)}(m, n)=O_{N \times N}, \\
& C r 2^{(k)}(m, n)=W c^{(k)}(m, n) * W c^{(k)}(m+1, n), 1 \leq m \leq J-1
\end{aligned}
$$

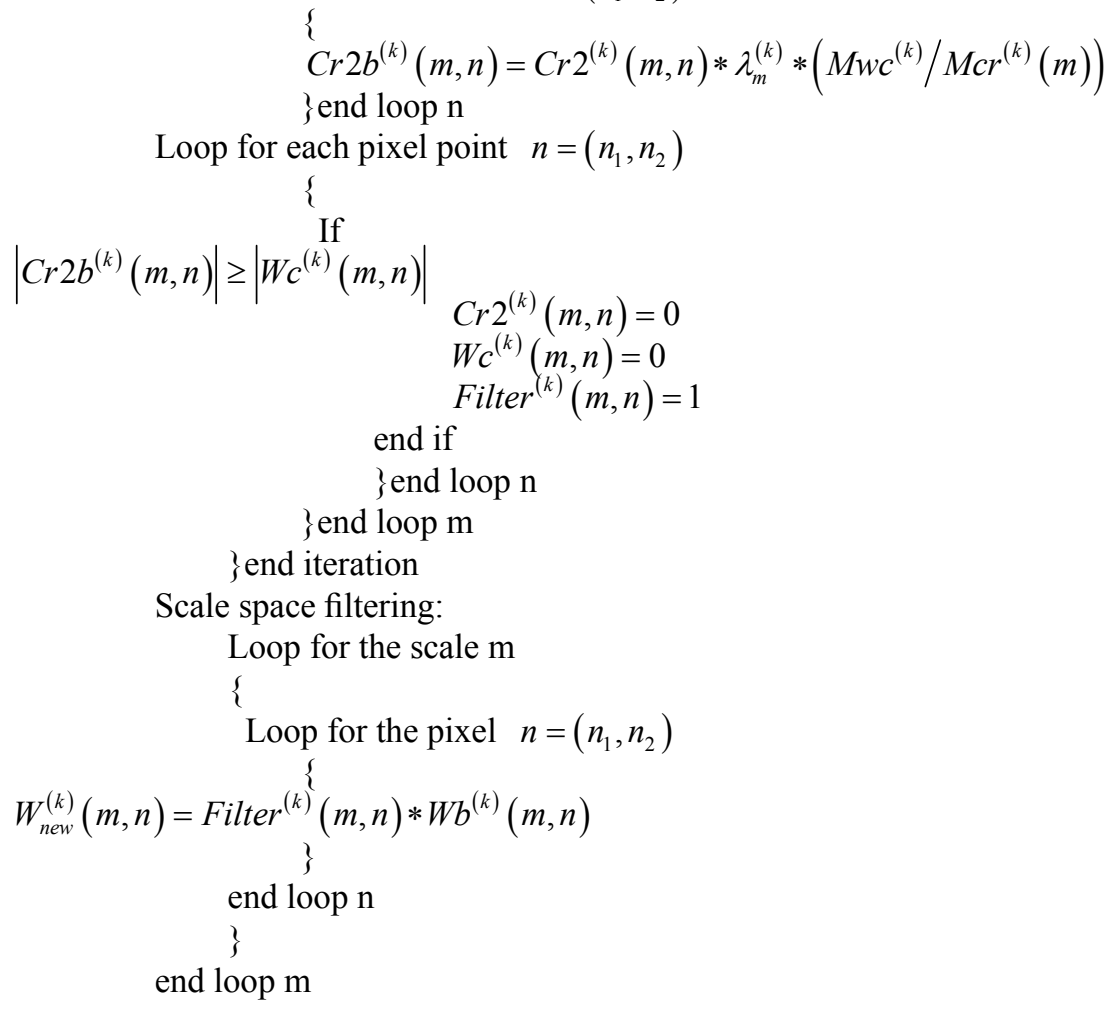

$$
\{
$$$$
C r 2 b^{(k)}(m, n)=C r 2^{(k)}(m, n) * \lambda_{m}^{(k)} *\left(M w c^{(k)} / \operatorname{Mcr}^{(k)}(m)\right)
$$

Step 4. Compute the wavelet coefficient at the maximum scale $J$ :

$$
W_{\text {new }}^{(k)}(J, n)=W^{(k)}(J, n)
$$

Step 5. Reconstruct the image from filtered wavelet data $\left\{W_{\text {new }}^{(k)}(j, n)\right\}_{1 \leq j \leq J}$ and $S_{2^{J}}(n)$

The reconstruction from the set $\left\{W_{\text {new }}^{(k)}(j, n), S_{2^{J}}(n)\right\}_{1 \leq j \leq J}$ through the inverse dyadic wavelet transform will yield the final filtered image. The inverse dyadic wavelet transform that we implemented in our technique uses a trous algorithm and the quadratic spline scaling functions and wavelets given in [18].

Now we give some comments on the choice of the number of iterations and weight parameter $\lambda_{m}^{(k)}$. We can design wavelet filtering iteration times and parameter $\lambda_{m}^{(k)}$ according to the user's request. For the number of iterations, when it is too small, we can not obtain a smooth estimate. If the number of iterations is too large, most of the edge information of reconstructed image will 
be eliminated. Thus we should choose a tradeoff between the number of iterations and the estimation of filtered image. It is well known that the Lipschitz exponents of image and noise are different. At the finer scales such as $2^{1}$ and $2^{2}$, the modulus maxima mainly produced by noise, while at coarser scale, most modulus maxima produced by image. So if we set the different value of $\lambda_{m}^{(k)}$ at the different scale $\mathrm{m}$, noise will be eliminated more effectively. In general, let parameter $\lambda_{m}^{(k)}$ be lager at the larger scale.

\section{Experimental Results}

We use Peak-Signal-Noise-Ratio (PSNR) and Root-MeanSquare-Error (RMSE) to evaluate restored results. PSNR and RMSE is defined by the following:

$$
\begin{gathered}
\operatorname{PSNR}(u, w)=10 \log 10 \frac{255^{2}}{\frac{1}{m n} \sum_{i, j}\left(u_{i, j}-w_{i, j}\right)^{2}} \\
\operatorname{RMSE}(u, w)=\sqrt{\frac{1}{m n} \sum_{i, j}\left(u_{i, j}-w_{i, j}\right)^{2}},
\end{gathered}
$$

where $w_{i, j}$ and $u_{i, j}$ denotes the pixel values of the processed and the original images respectively. Hardthresholding and soft-thresholding are widely used for denoising in image processing by many researchers. Therefore, in the following tests the hard-thresholding method, soft-thresholding method, Xu's method and Zheng's method will be used to compare with the dyadic wavelet transform filtering algorithm.

Example 1: When we use our filtering method to do denoising experiment for the above mentioned Lena image corrupted by additive noise, the restored result is Figure 2(a). If apply softthreshold, hard-threshold, Xu's method and Zheng's method to filter the noised image, the result is in Figures 2(b)-(e).

Table 1 presents the values of PSNR and RMSE for each of the schemes.

From all the five restored images, it is clear that our proposed method suppresses more noise while preserves more fine details and small structures in the image. In addition, from the values of PSNR and RMSE for restored image, our method increases the PSNR by $1-3 \mathrm{~dB}$ and reduce the RMSE 5 - 6.

Example 2: A texture image is used in the second test. It is added by the white Gaussian noise with the standard deviation $\sigma=30$. Use our filtering method, soft-thresholding method, hard-thresholding method, Xu's method and Zheng's method to process noised image, we can find that our method is also better than other four methods. Figure 3(a) is the original image, Figure 3(b) is the noised image. Figures 4(a)-(e) show the results processed by the five methods.

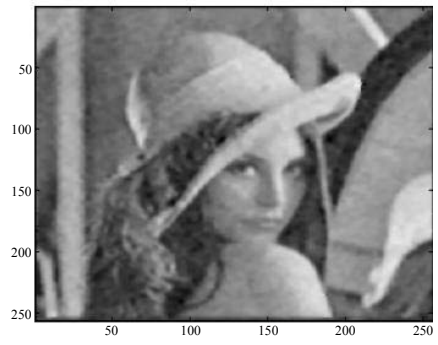

(a) Our method.

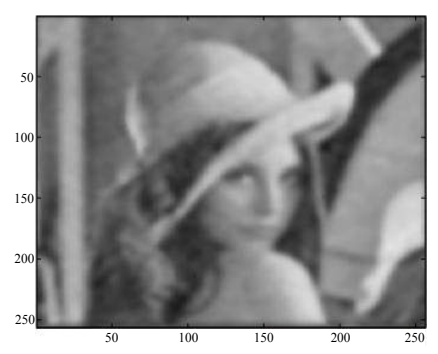

(b) Soft-threshold

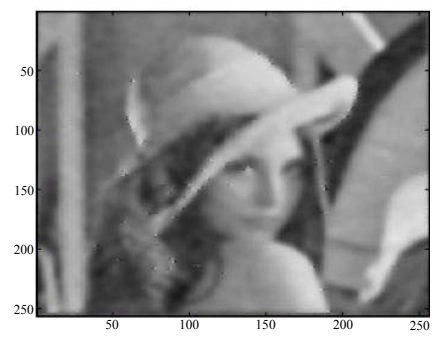

(c) Hard-threshold.

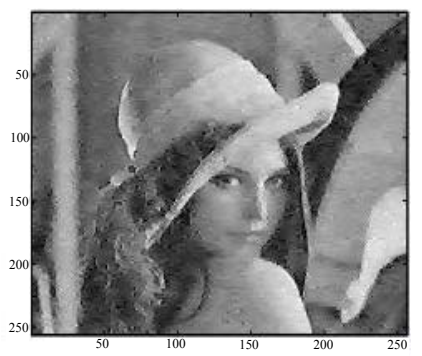

(d) Xu's Method.

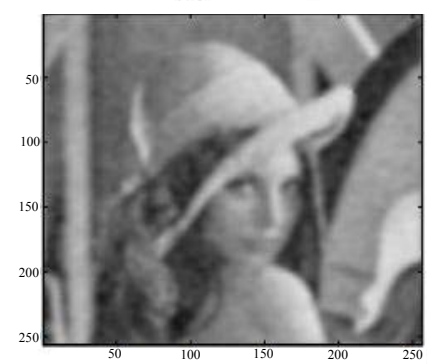

(e) Zheng's Method.

Figure 2. Filtered results. (a) Our method; (b) Soft-threshold; (c) Hard-threshold; (d) Xu's method; (e) Zheng's method.

Table 2 is the comparison of the values of PSNR and RMSE for restored images. 
Table 1. PSNR and RMSE for each of the schemes.

\begin{tabular}{cccccc}
\hline Method & $\begin{array}{c}\text { Our } \\
\text { method }\end{array}$ & $\begin{array}{c}\text { Soft- } \\
\text { threshold }\end{array}$ & $\begin{array}{c}\text { Hard- } \\
\text { threshold }\end{array}$ & $\begin{array}{c}\text { Xu's } \\
\text { method }\end{array}$ & $\begin{array}{c}\text { Zheng's } \\
\text { method }\end{array}$ \\
\hline PSNR & $25.2035 \mathrm{~dB}$ & $23.1125 \mathrm{~dB}$ & $22.4597 \mathrm{~dB}$ & $24.0583 \mathrm{~dB} 23.1079 \mathrm{~dB}$ \\
RMSE & 14.0076 & 17.8203 & 19.2111 & 15.9818 & 17.8297 \\
\hline
\end{tabular}
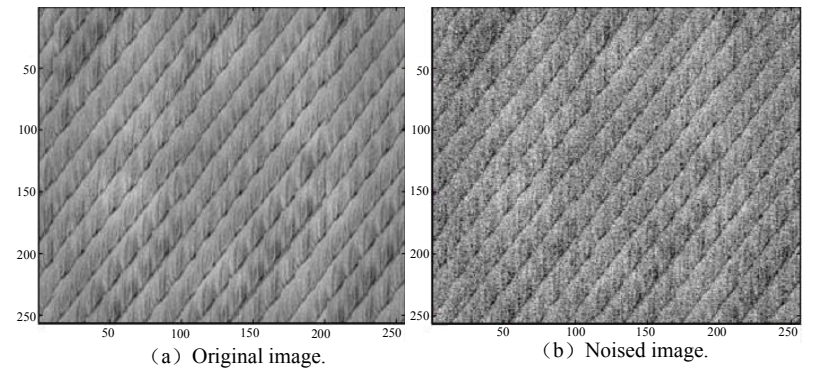

Figure 3. Texture image. (a) Original image; (b) Noised image.

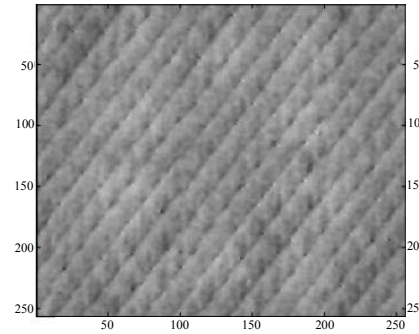

(a) Our method.

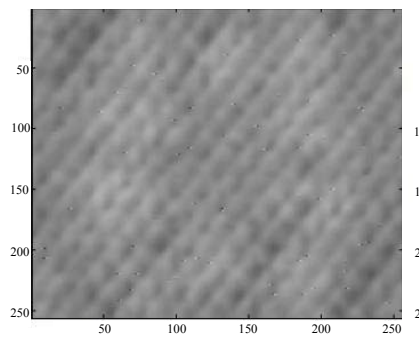

(c) Hard-threshold

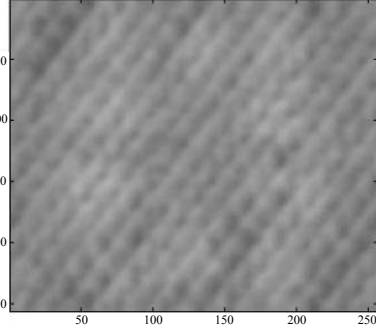

(b) Soft-threshold.

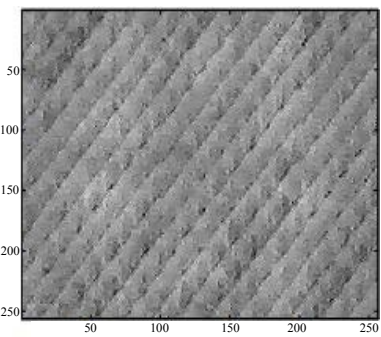

(d) Xu's Method

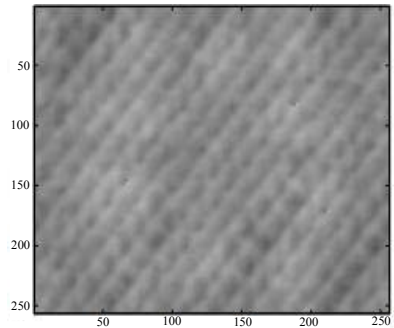

(e) Zheng's Method.

Figure 4. Results for the texture image. (a) Our method; (b) Soft-threshold; (c) Hard-threshold; (d) Xu's method; (e) Zheng's method.

Table 2. PSNR and RMSE for restored images.

\begin{tabular}{cccccc}
\hline Method & \multicolumn{2}{c}{ Our method $\begin{array}{c}\text { Soft-thresh Hard-thresh } \\
\text { old }\end{array}$} & $\begin{array}{c}\text { Xu's } \\
\text { old }\end{array}$ & $\begin{array}{c}\text { Zheng's } \\
\text { method }\end{array}$ & method \\
\hline PSNR & $24.1536 \mathrm{~dB}$ & $22.5607 \mathrm{~dB}$ & $22.5533 \mathrm{~dB}$ & $22.3974 \mathrm{~dB}$ & $22.7774 \mathrm{~dB}$ \\
RMSE & 15.8075 & 18.9892 & 18.9966 & 19.3488 & 18.5212 \\
\hline
\end{tabular}

From the results above, it is obvious that not only for visual quality of images, but also for quantitative evaluation of restored images, our method in texture image processing is still better than other four methods.

Example 3: We use a man image containing both a human face and some textures to do the third test. The challenge with this image is to keep both texture details and smooth transitions in the human face in the processing. We add the original image (Figure 5(a)) with the white Gaussian noise with $\sigma=30$, and get a noised image (Figure 5(b)). Figures 6(a)-(e) are the results obtained by five methods

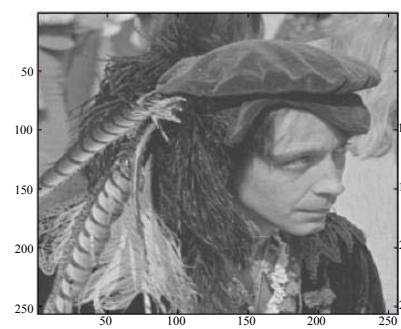

(a) Original image

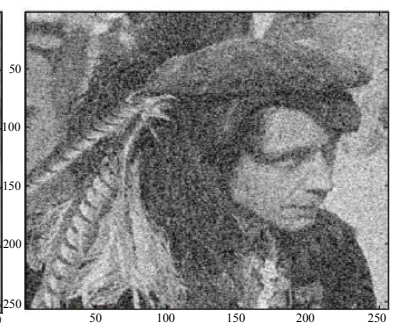

(b) Noisy image.
Figure 5. Man image. (a) Original image; (b) Noisy image.

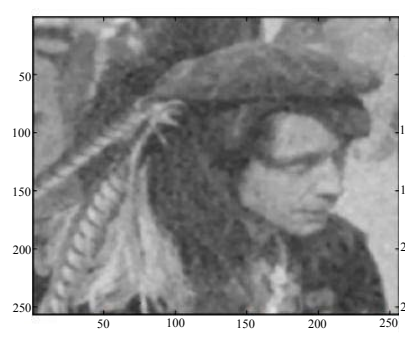

(a) Our method

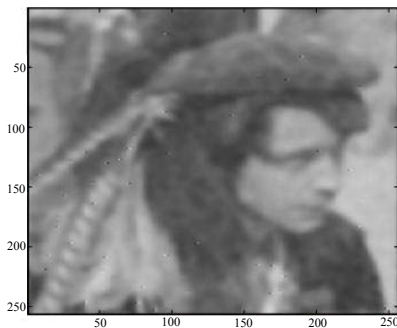

(c) Hard-threshold.

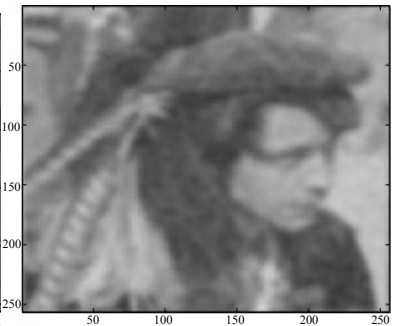

(b) Soft-threshold

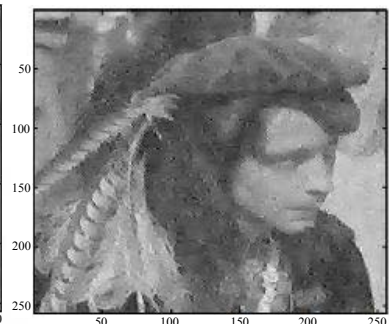

(d) Xu's Method.

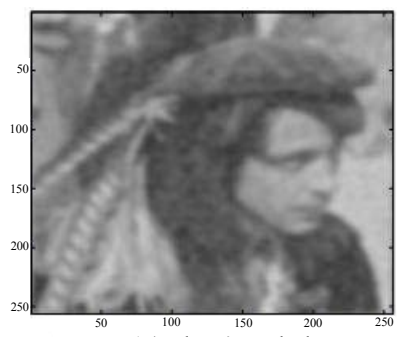

(e) Zheng's Method.

Figure 6. Results for man image. (a) Our method; (b) Softthreshold; (c) Hard-theshold; (d) Xu's method; (e) Zheng's method. 
Table 3 is the quantitative comparison among the five methods.

The results above reveal that our method not only maintain more texture details and smooth transitions in the face but also suppress more noise than other methods after processing. Additionally, our method can increase more PSNR and decrease RESE than other methods.

At last, we give some other types of images. And we only present results recovered by our method. A MR image (see Figure 7(a)) has been corrupted with white Gaussian noise $(\sigma=20)$ and become a noised image, see Figure 7(b). After the noised image has been processed with our method, we can see that our restoration scheme is able to maintain all important information and filter out much noise, see Figure 7(c).

Figures 8(a), (b) and (c) are an original building image, the noised image, and the processed result by our method. We can see that the recovered image can preserve more image edge details.

A fingerprint image is used in the last test. Figures 9(a)-(c) are the original image, the noised image with $\sigma=$ 15, and the recovered result with our scheme. From the visual quality, it is obvious that restored image is as good as the original one.

\section{Conclusions}

We have introduced the dyadic wavelet transform filtering

Table 3. Comparison of PSNR and RMSE for restored images.

\begin{tabular}{cccccc}
\hline Method & $\begin{array}{c}\text { Our } \\
\text { method }\end{array}$ & Soft-threshold Hard-threshold & $\begin{array}{c}\text { Xu's } \\
\text { method }\end{array}$ & $\begin{array}{c}\text { Zheng's } \\
\text { method }\end{array}$ \\
\hline PSNR & $\begin{array}{c}25.7523 \\
\mathrm{~dB}\end{array}$ & $23.8359 \mathrm{~dB}$ & $23.5585 \mathrm{~dB}$ & $\begin{array}{c}24.4842 \\
\mathrm{~dB}\end{array}$ & $\begin{array}{c}23.7759 \\
\mathrm{~dB}\end{array}$ \\
RMSE & 13.1500 & 16.3963 & 16.9284 & 15.2171 & 16.5099 \\
\hline
\end{tabular}

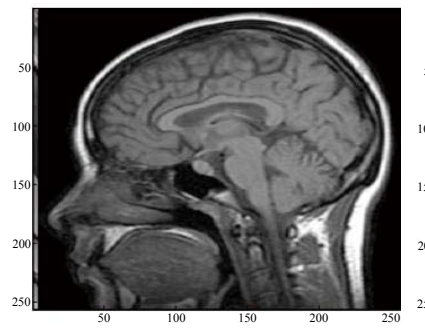

(a) Original image.

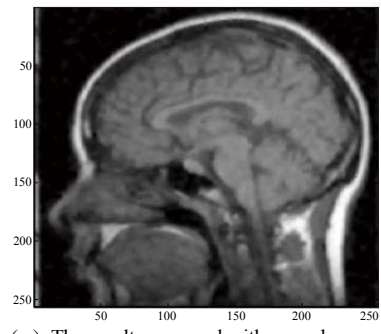

(c) The result recovered with our scheme.

Figure 7. MR image. (a) Original image; (b) Noised image with $\sigma=20$; (c) The result recovered with our scheme.

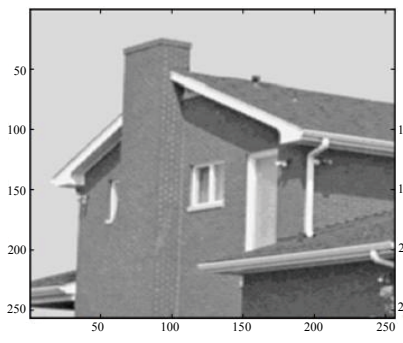

(a) Original image.

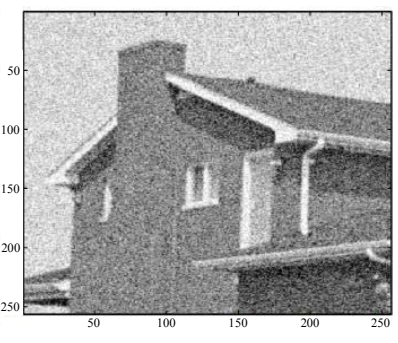

(b) Noised image with $\sigma=30$.

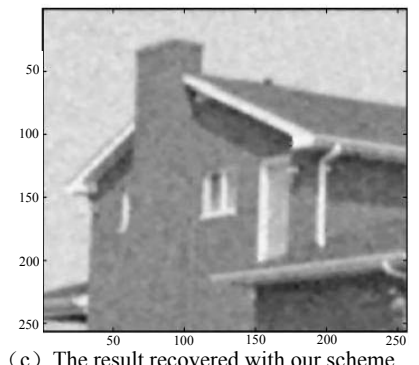

(c) The result recovered with our scheme.

Figure 8. Building image. (a) Original image; (b) Noisy image with $\sigma=30$; (c) The result recovered with our scheme.

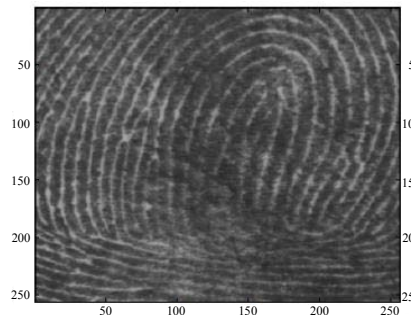

(a) Original image.

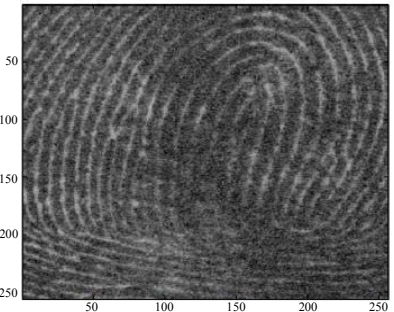

(b) Noised image with $\sigma=15$.

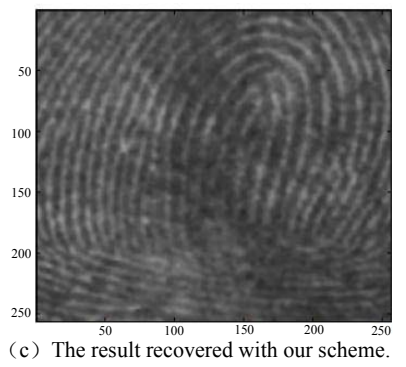

Figure 9. Fingerprint image. (a) Original image; (b) Noised image with $\sigma=15$; (c) The result recovered with our scheme.

technique for denoising in image processing. Our filtering algorithm is superior to soft-thresholding method, hardthresholding method, Xu's method and Zheng's method because important edge features in the wavelet transform domain are preserved while much noise is suppressed. The other filtering methods perform very poorly in image denoising because they tends to remove the high-frequency component exclusively, which yields smooth images and blurs the image edge features. 


\section{Acknowledgements}

This work was supported by the Key Project of Chinese Ministry of education (No. 109030), 382 Training Programme of CUC(G08382316), and Science Research Project of Communication University of China(XNL1003). The authors would like to thank the reviewers for very useful comments, which improved the manuscript significantly.

\section{REFERENCES}

[1] S. Mallat and S. Zhong, "Characterization of Signal from Multiscale Edges," IEEE Transactions on Pattern Analysis and Machine Intelligence, Vol. 14, No. 7, 1992, pp. 710-732. doi:10.1109/34.142909

[2] S. Mallat and W. L. Hwang, "Singularity Detection and Processing with Wavelets," IEEE Transactions on Information Theory, Vol. 38, No. 2, 1992, pp. 617-643. doi:10.1109/18.119727

[3] J. L. Starck and A. Bijaoui, Filtering and Deconvolution by the Wavelet Transform," Signal Processing, Vol. 35, No. 3, 1994, pp. 195-211. doi:10.1016/0165-1684(94)90211-9

[4] A. Bijaoui, "Wavelets, Gaussian and Wiener Filtering," Signal Processing, Vol. 82, No. 4, 2002, pp. 709-712. doi:10.1016/S0165-1684(02)00137-8

[5] P. L. Shui, "Image Denoising Algorithm via Best Wavelet Packet Base Using Wiener Cost Function," Institution of Engineering and Technology Image Processing, Vol. 1, No. 3, 2007, pp. 311-318.

[6] Y. Xu, J. B. Weaver, et al., "Wavelet Transform Domain Filters: A Spatially Selective Noise Filtration Technique," IEEE Transactions on Image Processing, Vol. 3, No. 6, 1994, pp. 747-758.

[7] C. Okechukwu and C. Ugweje, "Selective Noise Filtration of Image Signals Using Wavelet Transform," Imaging Measurement Systems, Vol. 36, No. 3-4, 2004, pp. 279-287.

[8] M. Nasri and H. Nezamabadi-pour, "Image Denoising in the Wavelet Domain Using a New Adaptive Thresholding
Function," Neurocomputing, Vol. 72, No. 4-6, 2009, pp. 1012-1025. doi:10.1016/j.neucom.2008.04.016

[9] Y. Zheng, D. B. H. Tay, et al., "Signal Extraction and Power Spectrum Estimation Using Wavelet Transform Scale Space Filtering and Bayes Shrinkage," Signal Processing, Vol. 80, No. 8, 2000, pp. 1535-1549. doi:10.1016/S0165-1684(00)00054-2

[10] Y. Leung, J. S. Zhang and Z. B. Xu, "Clustering by Scale-Space Filtering," IEEE Transaction on Pattern Analysis and Machine Intelligence, Vol. 22, No. 12, 2000 , pp. 1396-1410.

[11] J. Kalif, S. Mallat and B. Rouge, "Deonvolution by Thresholding in Mirror Wavelet Bases," IEEE Transaction on Image Processing, Vol. 12, No. 4, 2003, pp. 446457. doi:10.1109/TIP.2003.810592

[12] Y. F. Zheng and R. L. Ewing, "Feature-Based Wavelet Shrinkage Algorithm for Image Denoising," IEEE Transaction on Image Processing, Vol. 14, No. 12, 2005, pp. 2024-2039.

[13] Z. Y. Chen, X. P. Guo, X. L. Zhang, W. J. Cram and Z. W. Li, "A Novel Method for Analysis of Single Ion Channel Signal Based on Wavelet Transform," Computers in Biology and Machine, Vol. 37, No. 4, 2007, pp. 559-562. doi:10.1016/j.compbiomed.2006.08.006

[14] D. L. Donoho and I. M. Johnstone, "Ideal Spatial Adaption via Wavelet Shrinkage," Biometrika, Vol. 81, No. 3, 1994, pp. 425-435. doi:10.1093/biomet/81.3.425

[15] D. L. Donoho, "De-Noising by Soft-Thresholding," IEEE Transaction on Information Theory, Vol. 41, No. 3, 1995, pp. 613-627. doi:10.1109/18.382009

[16] M. Holschneider, R. Kronland-Martinet, J. Morlet and P. Tchamitchian, "Wavelets, Time-Frequency Methods and Phase Space, Chapter A Real-Time Algorithm for Signal Analysis with the Help of the Wavelet Transform," Springer-Verlag, Berlin, 1989, pp. 289-297.

[17] A. Witkin, "Scale Space Filtering," Proceedings of 8th International Joint Conference on Artificial Intelligence, Karlsruhe, 1983, pp. 1019-1022.

[18] S. Mallat, "A Wavelet Tour of Signal Processing," 2nd Edition, Academic Press, New York, 1999. 